\title{
OntoSIDES: Ontology-based student progress monitoring on the national evaluation system of French Medical Schools. ${ }^{\text {th }}$
}

\author{
Olivier Palombi ${ }^{\mathrm{a}, \mathrm{b}}$, Fabrice Jouanot ${ }^{\mathrm{c}}$, Nafissetou Nziengam ${ }^{\mathrm{c}, \mathrm{d}}$, Behrooz \\ Omidvar-Tehrani $^{\mathrm{c}}$, Marie-Christine Rousset ${ }^{\mathrm{c}, \mathrm{e}}$, Adam Sanchez ${ }^{\mathrm{c}}$ \\ ${ }^{a}$ Université Grenoble Alpes, LADAF, CHU Grenoble Alpes, F-38000 Grenoble, France \\ ${ }^{b}$ Université Grenoble Alpes, Grenoble INP, Inria, CNRS, LJK, F-38000, Grenoble, France \\ ${ }^{c}$ Université Grenoble Alpes, Grenoble INP, CNRS, Inria, LIG, F-38000, Grenoble, France \\ ${ }^{d}$ Yaoundé University, Cameroun \\ ${ }^{e}$ Institut universitaire de France, 75005, Paris, France
}

\begin{abstract}
We introduce OntoSIDES, the core of an ontology-based learning management system in Medicine, in which the educational content, the traces of students' activities and the correction of exams are linked and related to items of an official reference program in a unified RDF data model. OntoSIDES is an RDF knowledge base comprised of a lightweight domain ontology that serves as a pivot high-level vocabulary of the query interface with users, and of a dataset made of factual statements relating individual entities to classes and properties of the ontology. Thanks to an automatic mapping-based data materialization and rule-based data saturation, OntoSIDES contains around 8 millions triples to date, and provides an integrated access to useful information for student progress monitoring, using a powerful query language (namely SPARQL) allowing users to express their specific needs of data exploration and analysis. Since we do not expect end-users to master the raw syntax of SPARQL and to express directly complex queries in SPARQL, we have designed a set of parametrized queries that users can customize through a user-friendly interface.
\end{abstract}

Keywords: Learning Management System (LMS), Ontologies, RDF, rules,

\footnotetext{
This work has been partially supported by the LabEx PERSYVAL-Lab (11-LABX-002501) and the DUNE project SIDES 3.0 (ANR-16-DUNE -0002-02).
} 
SPARQL, e-Learning, Medicine

\section{Introduction}

SIDES is a french national e-learning platform for medical training online since 2013. Currently, the platform is used by all the French medical schools (34 over the country) for assessments and graduations. SIDES provides a shared 5 national training repository, filled by teachers who provide exam contents related to a national reference program organized by medical specialties and by items describing learning topics that are standardized across all universities through an official publication from the French Ministry of Higher Education in the socalled Bulletin Officiel [1. In this document, each named and defined item can in turn contain sub-items. The identifiers of the items and sub-items were used for tagging the questions of exams in the platform. Table 1 shows 2 items (out of 368) of the ECN program, the national ranking examination for accessing the third cycle (residency or postgraduate training) of Medical studies in France.

\begin{tabular}{|c|l|l|}
\hline Item ID & About item & Required skill(s) for the item \\
\hline 96 & Myasthenia & $\begin{array}{l}\text { - Diagnose a myasthenia } \\
\text { - Know treatments and plan the follow-up of the patient } \\
\end{array}$ \\
& & $\begin{array}{l}\text { - Know medicine dissuaded in the myasthenia } \\
\text { - Identify emergency situations and plan their coverage }\end{array}$ \\
\hline 262 & Urinary lithiasis & $\begin{array}{l}\text { - Diagnose an urinary lithiasis } \\
\text { - Argue the treatment and plan the follow-up of the patient }\end{array}$ \\
\hline
\end{tabular}

Table 1: Two items in the ECN program

SIDES is mainly used for a) academic ranking in medical schools and b) student self-assessment. In the last two years, more than 4 million self-assessment tests have been performed by students on SIDES. The adoption of computerbased exams allows the use of multimedia contents such as images, videos or sounds, and enables an automatic correction. 
SIDES is based on an operational PostgreSQL relational database $\ell^{1}$ and tions and full accessibility and availability.

SIDES graphical user interface (GUI) is limited to predefined interactions that do not fulfill the needs of its three types of end-users (students, teachers, and institutional administrators) that may have different demands.

With the mediation of the national coordinator for e-learning in Medicine (a co-author of this article) who is both teaching (as a Professor of Medicine) and responsible for making SIDES evolve in terms of pedagogical content and services, we have identified the following users requirements that are not fulfilled by SIDES for data analytics (categorized by types of users):

- Students requirements: students need to customize the analysis of their own performance in a given (or each) topic or specialty of the program ; in a competitive setting as in Medical studies, each student is also willing to analyse how his/her results (in a given or for each topic or specialty) would be compared with the overall results on the same topic or specialty of groups of students at the same level of study over a given period.

- Teachers requirements: teachers needs to monitor groups of students by analysing overall or individual results in a given topic or specialty; they have also expressed the need for fine-grained and domain-specific analysis of the discriminative power of the questions that have been answered by a given population of students during a given test of the national ranking examination.

- Institutional administrators requirements: At the institutional level, an overall vision is necessary to identify for instance items without (enough) associated questions. This type of information provides teachers with instructions on the coverage of the program during exams. It also allows

\footnotetext{
${ }^{1}$ https://www.postgresql.org
} 
to review the organization of the courses to improve the coverage of the program.

In this article, we describe OntoSIDES which is the ontology-based layer that we have developed on top of the SIDES database for facilitating the empowerment of SIDES end-users in data analytics, in particular to fulfill the above users requirements (i.e., students, teachers, and institutional administrators).

The remaining of the paper is organized as follows: in Section 2, we position our approach w.r.t. existing work ; in Section 3 we present our contributions ; finally, in Section 4 we conclude with some ongoing work and perspectives.

\section{Related work}

OntoSides is an implementation of the Educational Semantic Web vision initiated by several pioneering works [2, 3, 4] based on taking benefit of Semantic Web technologies for pushing forward Web-based educational environments. This vision has raised high expectations [5], and a lot has been done in this direction in the last decade (see [6] for a survey). However, a lot of things remain to be done for making this vision a reality [7. Despite some work on ontology extraction from existing course materials (e.g., [8]), or on modeling ontologies about learners (e.g., 9 , 10, 11, 12]), one of the main pending issues is the lack of fine-grained domain ontology covering the learning objects (courses, quiz) as well as the learning process (users, on-line actions) 13. The Learning Analytics Initiative [14] promotes high-level educational standards such as xAPI [15 for describing learning resources and students activities. Existing educational standards, such as xAPI, but also IMS Question and Test Interoperability specification (QTI) [16] or SCORM [17, aim at re-usability and interoperability

70 of learning content. In the existing learning analytics models, the assessment component is missing in all of them, except in the xAPI data model although insufficient [18, 19, 20].

However, these methods are not specialized enough for being used in practice by teachers in a specific domain such as Medicine. The concepts of a domain- 
specific e-learning ontology such as the one we developed in OntoSIDES can be declared as a specialization of high-level concepts of xAPI such as Activities, Responses or Interactions, or of IMS Tests and Questions model.

OntoSIDES follows the Open Educational Resource movement [21, 22], promoted by the Open University ${ }^{2}$ that assigns to every course a dedicated URI acting both as an identifier for the course on the Web, and as a way to relate it with useful information such as its level, its title, the topic and description of the course, and with other relevant open educational resources. To the best of our knowledge, OntoSIDES is the first ontology-based open education resource in Medicine, in which the educational content, the traces of students' activities and the correction of exams are linked and related to items of a official reference program in a unified data model.

Instead, from a system and methodological viewpoint, OntoSIDES follows an Ontology-based Data Access approach. Ontology-based Data Access (OBDA) 23] is a novel paradigm at the crossroad of Artificial Intelligence and Databases that has recently received an increasing interest because it enables end-users to ask their own (possibly complex) queries using a domain vocabulary they are familiar with. In contrast with standard relational databases for which the focus is the technical optimization of query evaluation by formatting data according to a pre-established schema, linking data to a domain ontology makes the data more understandable for users not familiar with database technologies. OBDA systems have a three-level architecture, constituted by an ontology, (relational) data sources, and mappings between the two. In the standard OBDA approach (called the virtual approach), the mappings are exploited to reformulate users' queries into effective queries evaluated over the data sources. In contrast, in OntoSIDES we exploit the mappings for data materialization. As a result, OntoSIDES supports full SPARQL 1.1, thus allowing to handle complex aggregated and counting queries. This is a big difference with the OBDA virtual approach for which the state-of-the-art query rewriting algorithms supports (union of)

\footnotetext{
${ }^{2}$ http://www.open.ac.uk
} 
conjunctive queries only.

Compared to educational data mining [24, the OBDA approach implemented in OntoSIDES makes learning analytics transparent and explainable to end-users (learners and teachers) which become actors of their data analytics.

\section{Results and discussion}

We have followed the Semantic Web [25] and Linked Data [26] standards for the semi-automatic and modular construction of OntoSIDES. OntoSIDES is a knowledge base comprised of a domain ontology that serves as a pivot high-level vocabulary of the query interface with users, and of a dataset made of factual statements relating individual entities to classes and properties of the ontology. Both the ontological statements and the factual statements are uniformly described in the RDF format [27, which makes possible to query OntoSIDES using the SPARQL query language [28]. RDF and SPARQL are standards recommended by W3C for the Semantic Web and Linked Data 3

For the semi-automatic construction of OntoSIDES we have chosen an expertbased methodology for building manually a lightweight domain ontology enriched with rules, and a OBDA mapping-based materialization approach for the automatic population of the ontology.

The incremental construction of the ontology, rules and the mappings was guided by the needs to express queries that could capture the users requirements highlighted in Section 1

Figure 1 shows the OBDA architecture of OntoSIDES. The data source layer corresponds to the SIDES relational database. The mapping layer is made of a set of mapping axioms (expressed using the Ontop syntax) that indicate how to transform relational data stored in SIDES into RDF data conform to the target RDFS schema specified in the ontology layer. The ontology layer is made of a set of RDFS statements and of a set of rules (expressed using the

\footnotetext{
${ }^{3}$ http://linkeddata.org/
} 


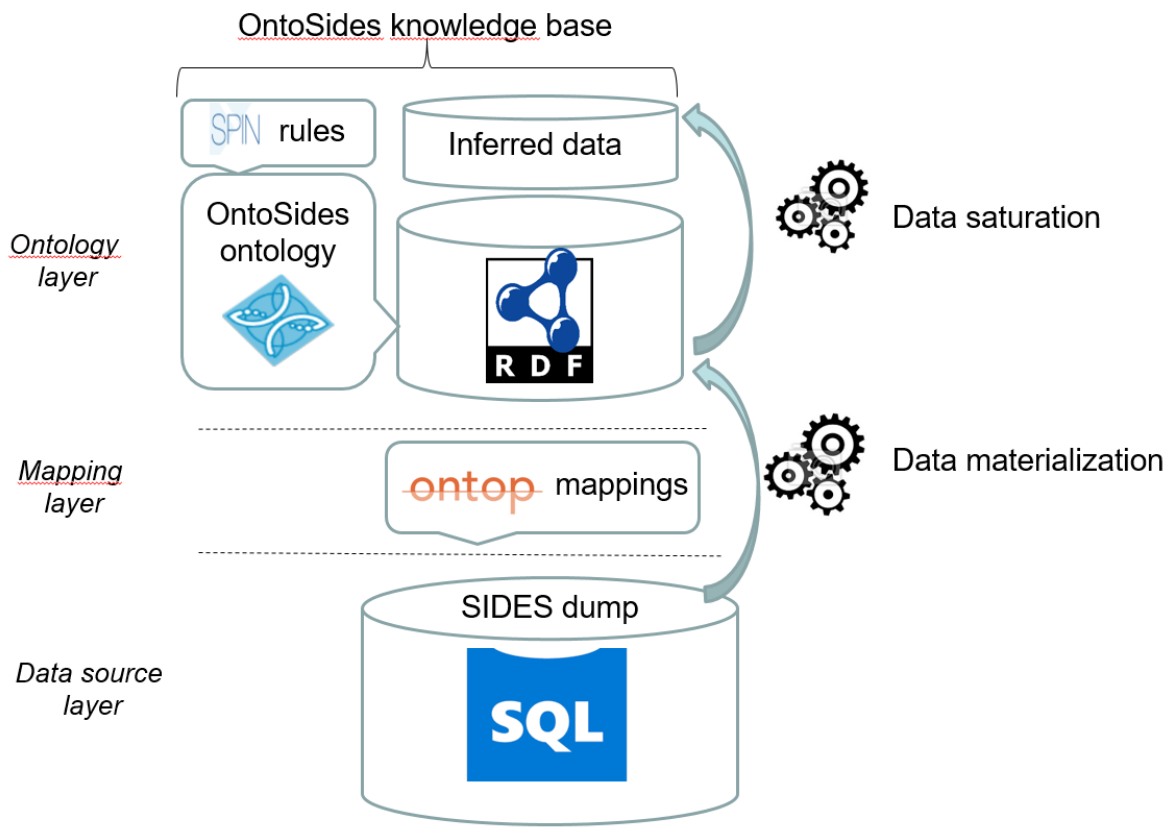

Figure 1: The OBDA architecture of OntoSIDES 
SPIN syntax) for defining classes and properties that are meaningful for the target end-users. Within the OntoSIDES knowledge base, we distinguish the OntoSIDES ontology and rules from the OntoSIDES dataset that is obtained by two distinct processes: data materialization, which is the process of exploiting the mappings in order to generate the RDF data corresponding to the instances of the classes and the properties defined in the OntoSIDES ontology ; and data saturation which, given the materialized data, is the process of making explicit the RDF data that can be logically derived from the RDFS statements and the SPIN rules.

We now summarize the results that have been produced and we provide some elements for their validation.

\subsection{Ontology and rules}

We have chosen the TOPBRAIDCOMPOSER software suite 29] to support an incremental methodology for building the OntoSIDES ontology, as TOPBRAIDComposer allows the definition of SPIN rules on top of RDF data and the application of these rules on RDF facts until saturation (i.e., until all the possible facts that can be inferred using the rules are obtained). RDF data equipped with rules capture most of OWL 30] constraints that are useful in practice, such as the transitivity or symmetry properties, as well as domain-specific rules with practical relevance for users in many domains of interest.

Using TopBraidComposer, the OntoSIDES ontology has been modelled by the national coordinator for e-learning in Medicine (a co-author of this article) in close cooperation with the other co-authors that are experienced in knowledge elicitation and ontology construction. Such an expert-based construction of the OntoSIDES ontology and rules has been facilitated by the unique position of the expert who has (i) a recognized and shared expertise on the organization and requirements of medical studies in France, (ii) a detailed technical knowledge on the SIDES database, and (iii) a previous experience in building ontologies in the related domain of anatomy [31, 32].

The resulting ontology consists of a taxonomy of 52 classes and a set of 50 
properties among which 3 are defined by rules. Figure 2 shows an extract of the class taxonomy (left) and of properties (right) as displayed by the TOPBRAIDComposer editor for the english version of the ontology.

Figure 2: Illustration of the English version of the ontology from TopBraidComposer

\begin{tabular}{|c|c|}
\hline 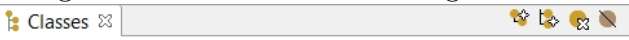 & Fi: Properties $\approx 3$ \\
\hline sides:sides_entity & E sides:correspond_to_a_question \\
\hline$\checkmark$ sides:action & E sides:did_not_check_expected_proposal \\
\hline - sides:action_to_answer & sides:done_by \\
\hline sides:city & E sides:done_during \\
\hline sides:content & E sides:ending_date_of_test \\
\hline > sides:educational_content & E sides:first_internship \\
\hline$\checkmark$ sides:evaluation_content & E sides:has_for_author \\
\hline$\sim$ sides:component_of_question & E sides:has_for_centre \\
\hline sides:proposal_of_answer & - sides:has_for_content \\
\hline - sides:statement_of_question & E sides:has_for_correction \\
\hline sides:evaluation_type & E sides:has_for_date \\
\hline - sides:progressive_clinical_case & E sides:has_for_description \\
\hline sides:set_of_isolated_questions & E sides:has_for_heading \\
\hline sides:question & E sides:has_for_hour \\
\hline sides:test & E sides:has_for_id \\
\hline sides:media & E sides:has_for_list_of_questions \\
\hline sides:picture & E sides:has_for_question \\
\hline sides:video & E sides:has_for_registrant \\
\hline sides:group & E sides:has_for_statement \\
\hline sides:institute & E sides:has_for_student_identification_number \\
\hline$\checkmark$ sides:person & E sides:has_for_title \\
\hline sides:administrative_agent & E sides:has_for_weight \\
\hline sides:student & E sides:has_for_weight_of_correction \\
\hline sides:teacher & E sides:has_for_year_of_study \\
\hline$\checkmark$ sides:referential_entity & E sides:has_rightly_ticked \\
\hline sides:cross_knowledge_entity & E sides:has_wrongly_ticked \\
\hline$\sim$ sides:ECN_referential_entity & E sides:is_an_item_of \\
\hline - sides:ECN_referential_item & E sides:is_an_subitem_of \\
\hline sides:ECN_referential_subitem & E sides:is_connected_to \\
\hline sides:ECN_teaching_chapter & [ sides:is_correct \\
\hline sides:speciality & - sides:is_enrolled_in_centreyear \\
\hline
\end{tabular}

The class taxonomy is built by successive refinements of 7 main classes which respectively denote:

- the set of possible actions of students when using the SIDES pedagogical online resources (denoted by the class sides:action in Figure2), 
- the types of pedagogical resources (training or evaluation) available in the SIDES platform (denoted by the class sides: content in Figure 2),

express that the property done_by serves to relate (identifiers of) actions extracted from SIDES log traces with (the identifiers of) the students who performed these specific actions. Some temporal properties such as starting_date_of_test and ending_date_of_test serve to associate the starting / ending time and date to the training tests taken by students.

- the set of reference items of the French educational program in Medicine (denoted by the class sides:referential_entity in Figure2), published by the French Ministry of Higher Education in Bulletin Officiel [1] and also used in SIDES as metadata,

- the sets of French cities, universities and medical schools (denoted by the classes sides:city and sides:institute in Figure 2 for which there are as many local SIDES platforms that are indexed in the central SIDES database,

- the set of milestones (years, periods of practical internships) to register and validate by students to get their diploma (denoted by the class sides:group in Figure 22, that are encoded in the SIDES database by specific identifiers,

- the categories of persons (students, academic staff, administrative staff) involved in medical studies (denoted by the class sides:person in Figure 2), that correspond to specific roles of users in the SIDES database.

The declaration of properties and their signature (using the pre-defined rdfs:domain and rdfs:range) completes the ontology by establishing how the instances of different classes can be related or described. For instance, the following RDF statements

$$
\text { (done_by rdfs:domain Action) and (done_by rdfs:range Student) }
$$


Some properties $p$ are defined by rules of the form IF Condition(?s) IF (?s $p$ $v$ ), where Condition(?s) is a conjunction of triple patterns expressing conditions for instances of the variable ?s to have $v$ as value for the property $p$. The different values of such properties are thus logically inferred by triggering the rules whose conditions can be matched with triples present in the dataset. For

not ticked atleast one correct answer) and one for adding the latter two numbers. The property has_for_result is then defined in function of the property $n b_{-} o f_{-} d i s c o r d a n c e$ by the following rules that compute the score (ranging from 0 to 1 ) of an action depending on its number of discordances. 

the instances of the property.

For the rules, their fine granularity allowed us to check that all the cases were covered to compute correctly all the possible values of the corresponding properties.

For assessing the global completeness of the ontology enriched with rules, our baseline was to check whether the classes and properties defined in the ontology and the rules enable to specify queries that capture the users' requirements that had been identified (and reported in Section 1). The parametrized queries shown in Section 3.3 provide a positive answer to this validation question. 


\subsection{Data materialization and saturation}

The OntoSIDES dataset is automatically constructed by a sequence of two processes that we explain below.

Mapping-based data materialization:

Using Ontop 33, we have defined as many mappings as classes and properregistered in Grenoble medical school, has resulted into 316,803 RDF triples that have been added to the OntoSIDES dataset as instances of the property sides:correspond_to_a_question. The result of the mapping-based data materi- 
It is worthwhile to emphasize that no personal data concerning students are extracted from SIDES. Instances of students in OntoSIDES are just represented by identifiers of the form sides : etu12402 (as in Example 1). There are no associated properties giving their name or any other personal information stored in the OntoSIDES dataset.

\section{Rule-based data saturation:}

Our rule-based approach is implemented by setting up the TOPBRAIDCOMPOSER inference mode to take into account RDFS and SPIN rules [34]. The inference mechanism is automatic and consists in applying the rules to the materialized dataset, in all the possible manners satisfying the conditions of the rules. For each possible instantiation of the variables (preceded by a question mark) appearing in a condition part of a given rule such that all its conditions are satisfied by explicit facts, the new facts corresponding to the (appropriately instantiated) conclusion of the rule are added. This saturation process is iterated as long as new facts can be generated. The termination is guaranteed by the form of the rules that are considered. They correspond to safe rules, also called Datalog rules [35], i.e., all the variables appearing in the conclusion of a rule also appears in the condition part.

305 This saturation process is done offline and the resulting set of inferred facts is added to the OntoSIDES dataset. The saturated version of the OntoSIDES dataset contains 7,962,247 triples. It has been processed in less than one hour on a standard notebook based on core i7 cpu, 16GB RAM and using Tobraid Composer 5 Rev 2.

\section{Methodology of validation}

The soundness and completeness of the ontoSIDES dataset, which is crucial for reliable data analytics, relies on the correctness of the mappings and of the rules that have generated it. Their verification is facilitated by the fact 
that each mapping and each rule targets a given class or property. Since the mappings and rules are specified in an explicit and declarative manner, their soundness is quite easy to check by a domain expert for whom the expressions in the source and target parts of the mappings (respectively, in the conditions and conclusions of the rules) are comprehensible. Understanding the expressions in the source part of the mappings requires in addition a good knowledge of the operational SIDES database since they are SQL queries expressed in the schema of the SIDES database. For helping the expert to validate and to complete the mappings if necessary, we use SPARQL queries to check whether properties known to be mandatory for a given class (e.g., the property done_by for the class Action) are valued for every instance of the class. If this is not the case, the corresponding mapping is incomplete and must be modified accordingly.

We follow a similar approach to check the completeness of a set of rules for defining a given property, like the 4 rules above for defining the property has_for_result that should assign a value to all the answering actions in the dataset. This can be seen as a cross-validation of the rules and the data.

\subsection{Parametrized queries}

The OntoSIDES knowledge base provides an integrated access to useful information extracted from different data sources, with a powerful query language (namely SPARQL) allowing users to express their specific needs of data exploration and analysis. Through SPARQL queries, a student can explore the set of reference items for which he/she has not trained yet, or launch a comparative analysis of his/her progress on a specific part of the program. Also a teacher may be interested in analyzing the strengths and weaknesses of his/her group of students compared to other groups at the same study level in other universities.

Example1 provides the SPARQL syntax of a query to retrieve the list of ECN

reference items for which a given student (sides: etu12402) has not answered any related question.

\section{Example 1.}


SELECT ?item WHERE \{?item a sides:ECN_referential_item .

FILTER NOT EXISTS \{?q sides:is_linked_to ?item.

?a sides:done_by sides:etu12402.

?a sides:corresponds_to_question ?q\}\}

SPARQL can also be used as a declarative tool of tabular data preparation for advanced data analytics or visualization.

Full SPARQL (i.e., SPARQL 1.1) allows to specify on-demand calculation of simple statistical summaries of data blocks thanks to so-called aggregation queries. An aggregation query enables a user to declare data blocks of interest and numbers about these blocks that she wants to calculate based on aggregate functions. Such aggregation queries can be used to calculate distributions of numerical data that are computed on-the-fly by grouping facts in the knowledge base, such as grouping the questions by entity of the program to count them, or to compute the average marks obtained by (some groups of) students.

The distinguishing point with standard relational databases (or with Excel sheets) is that grouping can be defined on properties that are not explicitly instantiated but whose values must be inferred. In the setting of RDF knowledge bases, it is worth to outline that, without inference, the statistics obtained by evaluating aggregation queries may be incorrect even if they are correctly specified by users. For instance, Example 2 shows a simple SPARQL aggregation query that counts, for each entity of the program, the number of questions related to it.

Example 2.

SELECT ?entity ((COUNT (DISTINCT ?q)) AS ?nbRelatedQuestions)

WHERE $\{$ ?q rdf:type sides:question.

?q sides:is_linked_to ?entity.

?entity rdf:type sides:referential_entity

\} GROUP BY ?entity

If it is evaluated against OntoSIDES before saturation, it will return an empty answer because the class sides:question has no explicit instance since each 
specific question appears in fact as an instance of one of the sides:question's subclasses sides: QMA (denoting questions with multiple answers) or sides : QUA (denoting questions with unique answers). For the sides:referential_entity class, similarly, its (implicit) instances are in fact categorized as medical specialties (grouped as instances of the subclass sides:speciality), or pieces of transverse knowledge (instances of the sides:cross_knowledge_entity subclass), or items of the ECN program (denoted by the sides:ECN_referential_entity subclass). When evaluated after the saturation of OntoSIDES, it will provide a table with correct answers: a line per entity having related questions with the right number of related questions in each line.

We do not expect end-users to master the raw syntax of SPARQL and to express directly complex queries in SPARQL. In fact, we have designed a set of parametrized queries that users can customize through a user-friendly interface available at http://ontosides.univ-grenoble-alpes.fr/. To achieve realtime answering for multi-user access the Ontology layer uses the Virtuoso triplestore in version 7 on a server computer based on Intel Xeon 8 cpu E5-2699 @ 2.20Ghz with 16GB RAM.

We follow the categorization of the users' needs depicted in Section 1 to illustrate how these parametrized queries evaluated on top of the OntoSIDES knowledge base fulfill them.

\section{Student-centered analyses}

Figure 3 shows the interface for students who can choose to launch one of the six queries in the left column that will be parametrized by his/her identifier (sides:etud12402 in the example). The chart in the right side of the Figure shows the result returned to the first query for that student, i.e., the average results obtained per specialty by the student (to all the questions he/she answered related to this specialty), compared to the overall average obtained per specialty by the whole group of active students.

Based on these results, it is easy to see that this student has an excellent grade profile with very high scores above average in all specialities. 


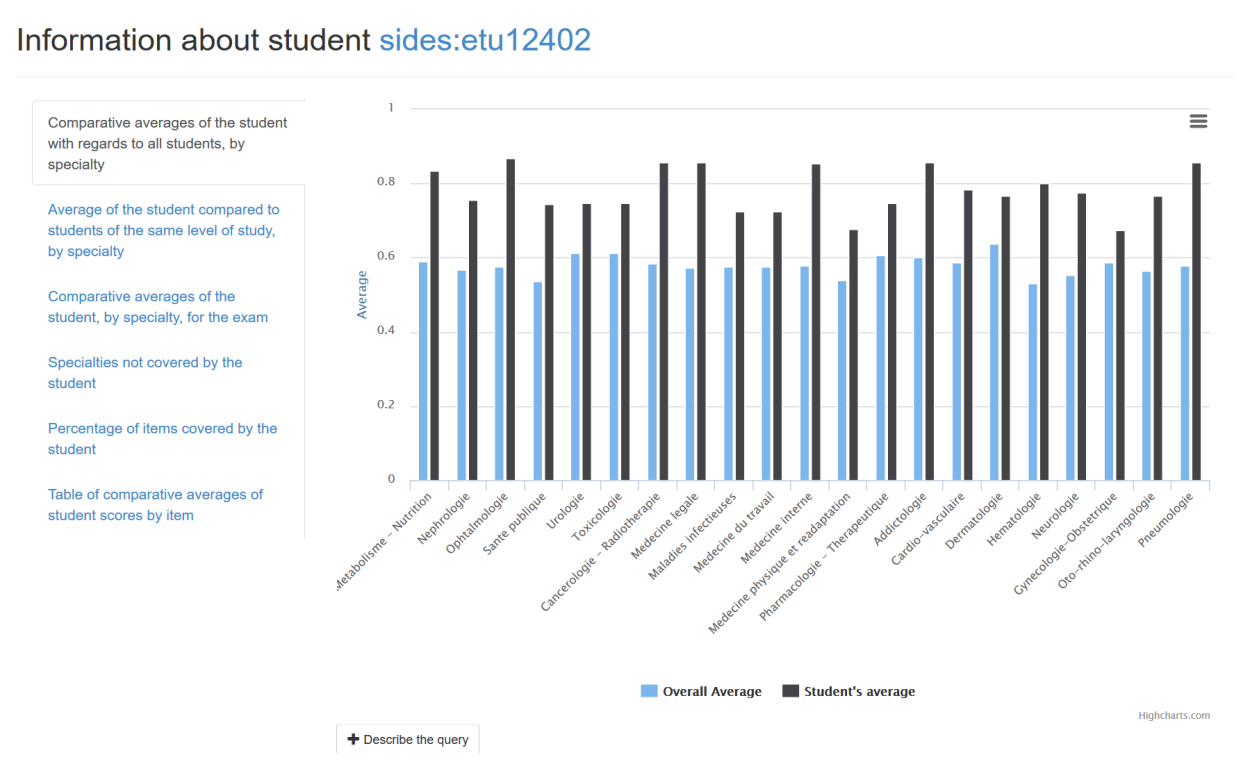

Figure 3: Parametrized queries for students

In contrast, Figure 4 exhibits the results of a student with a grade profile very close to that of the overall average. Personalized recommendations can however 405 be given to him/her: he/she must focus on neurology, hepato-gastroenterology, gynecology and pediatrics if she wishes to improve her skills in these specialties. 


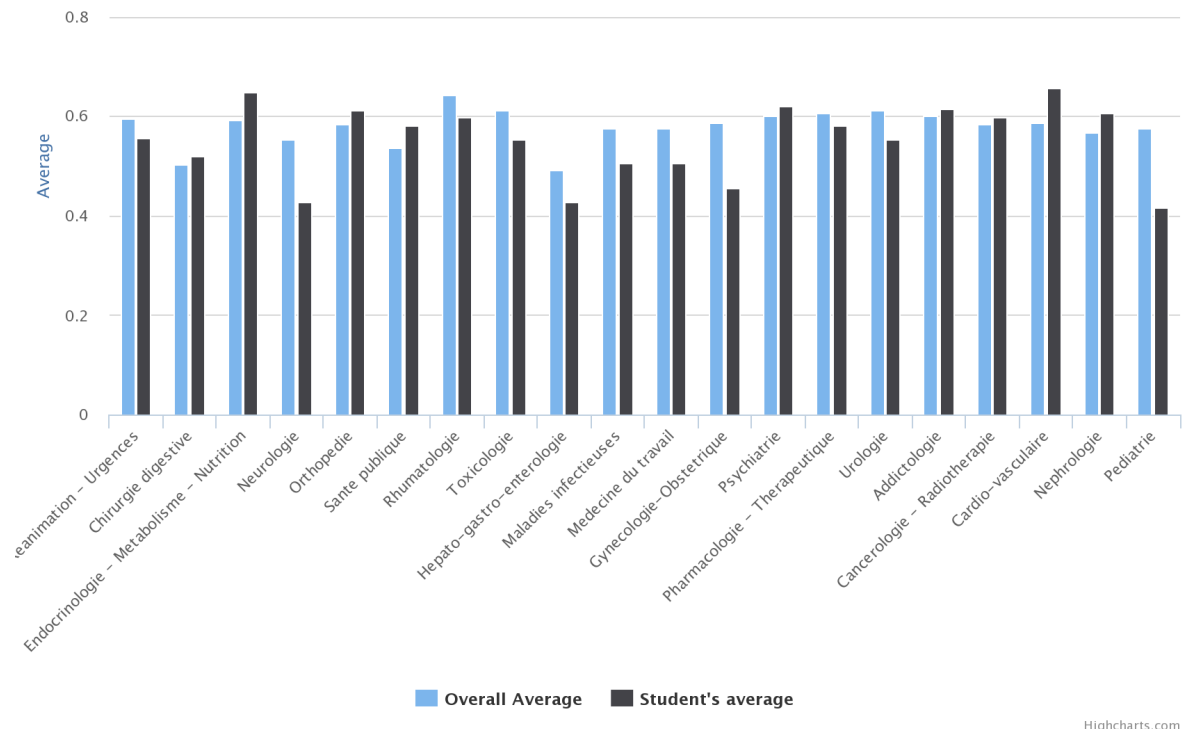

Figure 4: Results of an average student with small variations by specialties

Finally, Figure 5 exhibits the results of a student who is in trouble since he/she is below average in most of the specialties, except in cardiology, anesthesia and intensive care. A personalized procedure can be used to motivate 410 him/her to work in the other specialties. 


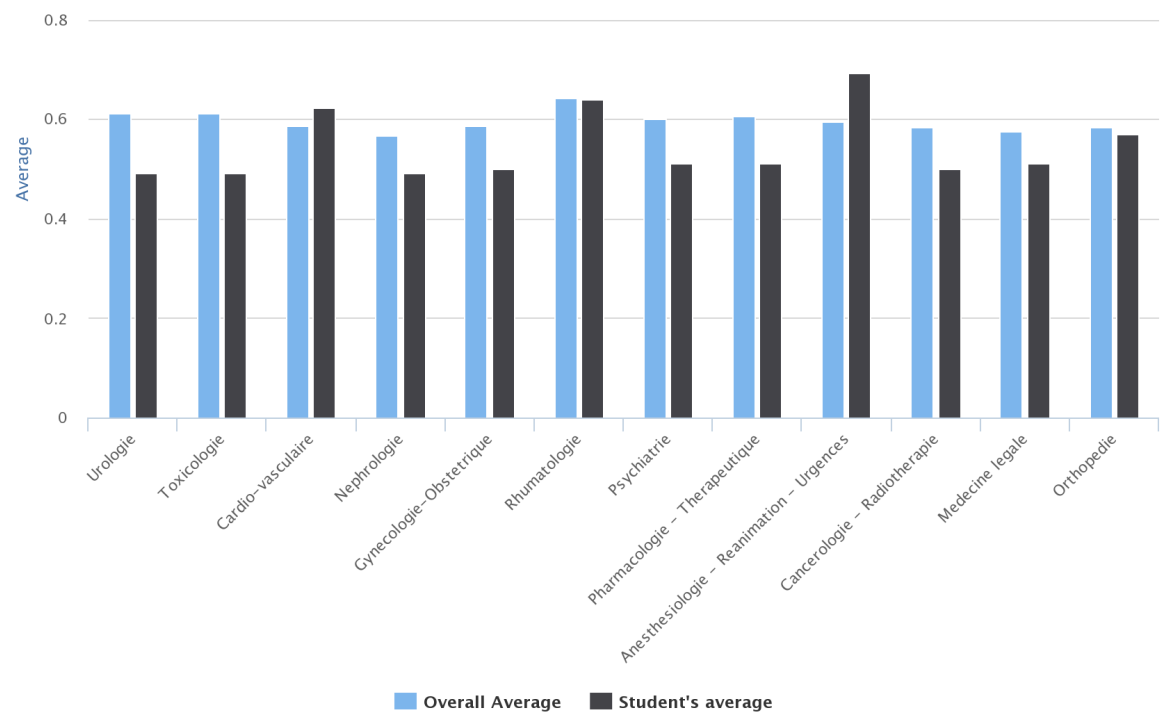

Figure 5: Results of a below average student in almost all the specialities

There exists plenty of other student-centered analyses. For instance, the query of Example 1 is an instantiation of a parametrized query that gets the list of items not covered by a given student. It is also possible to deepen and detail the analysis of average scores of one student per item to identify items to work specifically.

\section{Teacher-centered analyses}

Teachers may be interested by monitoring each student individually (as illustrated previously), or a group of students. They can also have more complex needs that require the evaluation of a chain of queries. This is the case for instance for finding which questions of a given test were able to discriminate between the students who took the test. This is a crucial criteria for adjusting the weight of questions for the final ranking of the annual national classification examination. For a given test, a question is judged discriminative by teachers if there is a significant difference between the average marks obtained for this 

the students of Group3. Figure 6 shows the top-4 most discriminative questions for a given test, according to the above specification.

For sides:eval52749 in sides:epreuve43969

\begin{tabular}{lllll}
$\mathbf{f}$ Show the query & & & \\
\cline { 1 - 3 } $\mathbf{q}$ & AverageQuestionGroup1 & AverageQuestionGroup3 & Difference \\
\hline sides:q406589 & 0.8 & 0.181818181818182 & 0.618182 \\
\hline sides:q406588 & 0.961538461538462 & 0.491666666666667 & 0.469872 \\
\hline sides:q406586 & 0.707692307692308 & 0.284615384615385 & 0.423077 \\
\hline sides:q406582 & 0.884615384615385 & 0.469230769230769 & 0.415385 \\
\hline
\end{tabular}

Figure 6: Comparison between the top and bottom student groups of their result for three questions of a given test

\section{Administrator-centered analyses}

At the institutional level, an overall vision is necessary to assess the global organization of the training activities. We have designed a set of parametrized queries (accessible by clicking "Management queries" in the banner of the Web interface) that provide some useful statistics for this. For instance, Figure 7 
provides the list of items in the ECN program without associated questions.

\begin{tabular}{ll} 
item & intituleltem \\
\hline sides:item_75 & Addiction aux medicaments psychotropes (benzodiazepines et apparentes) (voir item 319) \\
\hline sides:item_87 & Alteration de la fonction auditive (voir item 127) \\
\hline sides:item_79 & Alteration de la fonction visuelle (voir item 127) \\
\hline sides:item_133 & Anesthesie locale, locoregionale et generale \\
\hline sides:item_80 & Anomalie de la vision d'apparition brutale \\
\hline
\end{tabular}

Figure 7: Items without associated question

By clicking the button "Describe the query", users can see the SPARQL queries that compute the results, as shown in Figure 8 . Such functionality can help users to make sense of the computations that are done and to modify themselves the query to adjust the desired computation.

\section{Ontosides Management queries - Data about a student Groups comparison}

\section{Items without associated question}

+ Describe the query

Repository items with their titles that have no associated questions.

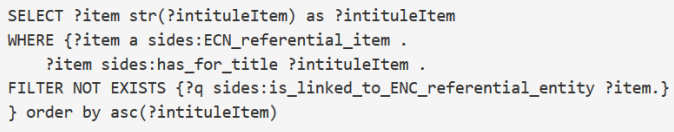

Results

Figure 8: The SPARQL query that computes the list of items without associated question 


\section{Conclusion and Perspectives}

OntoSIDES is a proof of concept for the feasability of an ontology-based

450 tology provides a query interface for end-users to interact with data stored about their learning activities related to a given educational program. This enables end-users to receive personalized feedback and recommendations, and to become the major actors of their own progress monitoring and training. Our approach is centered on an ontology which serves as a pivot vocabulary relevant both for students, teachers and institutional administrators involved in education for a given discipline. The construction of the OntoSIDES ontology has been facilitated by the fact that Medicine is a discipline for which teaching objectives and evaluations are well codified standards shared by a whole community.

OntoSIDES has been a decisive step in setting up the ongoing SIDES 3.0 project that is conducted under the authority of UNESS (https://www.uness.fr/), Université Numérique en Santé et en Sport. The methodology and architecture set up for the version of OntoSIDES described in the current article have shown their robustness since the mapping-based data materialization of the OntoSIDES dataset has scaled up to 1,5 billions of triples just by applying it to a full dump of the SIDES database (corresponding to the activities of 65,000 students over several years). In SIDES 3.0, the goal is to remove the current operational SIDES database and to replace it by an OntoSIDES evolving knowledge graph.

The methodology that we developed is not specific to Medicine. It can be reused for enriching or building learning management systems in other disciplines. The effort is depending mainly on the existence of a standard referential for the educational program in the target discipline.

Our ongoing work aims at palliating the incompleteness of the SIDES database and thus of OntoSIDES in which explicit links are missing between questions and items of the programme or specialities for a significant fraction of the questions. We are investigating how to enrich OntoSIDES by automatic links discovery 
using text mining techniques.

\section{References} http://www .enseignementsup-recherche.gouv.fr/pid20536/ bulletin-officiel.html?cid_bo=71544\&cbo=1, bulletin Officiel $n^{o}$ 20, May 16th (2013).

[2] V. Devedzic, Education and the Semantic Web, Int. J. Artif. Intell. Ed. and Practicing the Future of Education, Journal of Interactive Media in Education 2004 (1) (2004) Art. 1. doi:10.5334/2004-1. URL http://jime.open.ac.uk/articles/10.5334/2004-1/

[4] L. Aroyo, D. Dicheva, The New Challenges for E-learning: The Educational Semantic Web, Educational Technology \& Society 7 (2004) 59-69.

[5] P. Miranda, P. Isaias, C. J. Costa, E-Learning and web generations: Towards Web 3.0 and E-Learning 3.0, in: International Proceedings of Economics Development and Research, Vol. 81, IACSIT Press, 2014, p. 92.

[6] K. Krieger, D. Rosner, Linked data in e-learning: A survey, Semantic Web Journal 1.

[7] I. A. Bogoslov, Future Research Directions on Web-Based Educational Systems, in: R. Ortean, C. Ogrean, S. C. Mrginean (Eds.), Innovative Business DevelopmentA Global Perspective, Springer Proceedings in Business and Economics, Springer International Publishing, 2018, pp. 9-20.

[8] M. Hatala, D. Gaevi, M. Siadaty, J. Jovanovi, C. Torniai, Can Educators Develop Ontologies Using Ontology Extraction Tools: An End-User Study, 
in: U. Cress, V. Dimitrova, M. Specht (Eds.), Learning in the Synergy of Multiple Disciplines, Springer Berlin Heidelberg, Berlin, Heidelberg, 2009, pp. $140-153$.

[ [9] M. R. F. Sani, N. Mohammadian, M. Hoseini, Ontological Learner Modeling, Procedia - Social and Behavioral Sciences 46 (2012) 5238 5243. doi:https://doi.org/10.1016/j.sbspro.2012.06.416.

[10] M. Rani, R. Nayak, O. P. Vyas, An ontology-based adaptive personalized e-learning system, assisted by software agents on cloud storage, KnowledgeBased Systems 90 (2015) 33-48. doi:10.1016/j.knosys.2015.10.002 URL http://ww.sciencedirect.com/science/article/pii/ S0950705115003779

[11] M. Rani, K. V. Srivastava, O. P. Vyas, An ontological learning management system, Computer Applications in Engineering Education 24 (5) (2016) 706-722. doi:10.1002/cae.21742. URL https://onlinelibrary.wiley.com/doi/abs/10.1002/cae. 21742

[12] M. Kultsova, A. Anikin, I. Zhukova, A. Dvoryankin, Ontology-based learning content management system in programming languages domain, in: Proceeding of the First Conference on Creativity in Intelligent Technologies and Data Science, Communications in Computer and Information Science, 2015, pp. 767-777. doi:10.1007/978-3-319-23766-4.

[13] R. D. Morris, Web 3.0: Implications for Online Learning I, TechTrends 55 (1). doi:https://doi.org/10.1007/s11528-011-0469-9. URL https://mafiadoc.com/web-30-implications-for-online-learning-i_ 5a262d341723dd4b03de09b5.html

530 [14] The Apereo Learning Analytics, https://www.apereo.org/communities/ learning-analytics-initiative. 
[15] The Experience API (xAPI) for learning technology, https:// experienceapi.com/

[16] The IMS Question and Test Interoperability specification, https://www. imsglobal.org/question/index.html.

[17] Sharable Content Object Reference Model (SCORM), https://scorm. $\mathrm{com} /$.

[18] A. Nouira, L. Cheniti-Belcadhi, R. Braham, An enhanced xapi data model supporting assessment analytics, in: Knowledge-Based and Intelligent Information \& Engineering Systems: Proceedings of the 22nd International Conference KES-2018, Belgrade, Serbia, 3-5 September 2018., 2018, pp. 566-575. doi:10.1016/j.procs.2018.07.291.

URL https://doi.org/10.1016/j.procs .2018.07.291

[19] A. Nouira, L. Cheniti-Belcadhi, R. Braham, A semantic web based architecture for assessment analytics, in: 29th IEEE International Conference on Tools with Artificial Intelligence, ICTAI 2017, Boston, MA, USA, November 6-8, 2017, 2017, pp. 1190-1197. doi:10.1109/ICTAI.2017.00181. URL https://doi.org/10.1109/ICTAI.2017.00181

[20] A. Nouira, L. Cheniti-Belcadhi, R. Braham, An ontological model for assessment analytics, in: Proceedings of the 13th International Conference on Web Information Systems and Technologies, WEBIST 2017, Porto, Portugal, April 25-27, 2017, 2017, pp. 243-251. doi:10.5220/ 0006284302430251 .

URL https://doi.org/10.5220/0006284302430251

${ }_{555}$ [21] Open Educational Resource Handbook, http://wikieducator.org/OER_ Handbook.

[22] D. Mouromtsev, Mathieud'Aquin (Eds.), Open Data for Education: Linked, Shared and Reusable Data for Teaching and Learning, Springer, 2016. doi:10.1007/2F978-3-319-30493-9. 
[23] A. Poggi, D. Lembo, D. Calvanese, G. D. Giacomo, M. Lenzerini, R. Rosati, Linking data to ontologies, Journal f Data Semantics 10 (2008) 133-173.

[24] C. Romero, S. Ventura, M. Pecheniszkiy, R. Baker (Eds.), Handbook of Educational Data Mining, CRC Press, 2010.

[25] D. Allemang, J. Hendler, Semantic Web for the Working Ontologist: Mod-

[26] T. Heath, C. Bizer, Linked Data : Evolving the Web into a Global Data Space, Morgan and Claypool, 2011.

[27] W3C, RDF Description Format, http://www.w3.org/TR/rdf-concepts/ (2004).

570

[28] W3C, SPARQL 1.1 Overview, https://www.w3.org/TR/ sparql11-overview/ (2013).

[29] Top Braid Composer from TopQuadrant software, http://www . topquadrant.com/.

[30] W3C, Web Ontology Language (OWL), http://www.w3.org/2004/OWL/

[31] O. Palombi, F. Ulliana, V. Favier, J.-C. Leon, M.-C. Rousset, My corporis fabrica: an ontology-based tool for reasoning and querying on complex anatomical models, Journal of Biomedical Semantics (JOBS 2014) 5 (20).

[32] P.-Y. Rabattu, B. Masse, F. Ulliana, M.-C. Rousset, D. Rohmer, J.C. Leon, O. Palombi, My corporis fabrica embryo: An ontology-based 3d spatio-temporal modeling of human embryo development, Journal of Biomedical Semantics (JOBS 2015) 6 (36).

[33] D. Calvanese, B. Cogrel, S. Komla-Ebri, R. Kontchakov, M. R. Davide Lanti, M. Rodriguez-Muro, G. Xiao, Ontop: Answering sparql queries

$585 \quad$ over relational databases, Semantic Web Journal (SWJ 2017). 
[34] W3C, SPIN Overview and Motivation, https://www.w3.org/ Submission/spin-overview/ (2011).

[35] S. Abiteboul, R. Hull, V. Vianu, Foundations of Databases, AddisonWesley, 1995. 